\title{
Detection of non-Gaussian signatures in the VIRMOS-DESCART lensing survey*
}

\author{
F. Bernardeau ${ }^{1}$, Y. Mellier ${ }^{2,3}$, and L. van Waerbeke ${ }^{2,4}$ \\ 1 Service de Physique Théorique, CE de Saclay, 91191 Gif-sur-Yvette Cedex, France \\ 2 Institut d'Astrophysique de Paris, 98bis boulevard Arago, 75014 Paris, France \\ 3 Observatoire de Paris, LERMA, 61 avenue de l'Observatoire, 75014 Paris, France \\ 4 Canadian Institute for Theoretical Astrophysics, 60 St George St., Toronto, Ont. M5S 3H8, Canada
}

Received 27 February 2002 / Accepted 14 May 2002

\begin{abstract}
We have detected non-Gaussian signatures in the VIRMOS-DESCART weak lensing survey from a measurement of the three-point shear correlation function, following the method developed by Bernardeau et al. (2002). We obtain a $2.4 \sigma$ signal over four independent angular bins, or equivalently, a $4.9-\sigma$ confidence level detection with respect to measurements errors on scale of about 2 to 4 arcmin. The amplitude and the shape of the signal are consistent with theoretical expectations obtained from ray-tracing simulations. This result supports the idea that the measure corresponds to a cosmological signal due to the gravitational instability dynamics. Its properties could be used to put constraints on the cosmological parameters, in particular on the density parameter of the Universe, but the error level as well as the cosmic variance are still too large to permit secure conclusions.
\end{abstract}

Key words. cosmology - gravitational lensing - large scale structure

\section{Introduction}

The large-scale structure of the Universe are expected to form from the gravitational growth of initial density perturbations obeying Gaussian statistics. As the Universe expands and the perturbations grow, non-Gaussian features are expected to emerge in the density field due to gravitational dynamics. These features can be characterized with perturbation theory calculations, which allow to compute for instance the skewness, third moment of the local density probability distribution function (Peebles 1980; Fry 1984; Bernardeau 1992). The reduced skewness of the density field has been found to be quite insensitive to the variance and the cosmological parameters, $\Omega_{\mathrm{m}}$ (Juszkiewicz et al. 1992; Bernardeau 1994). In contrast, weak lensing surveys are sensitive to $\Omega_{\mathrm{m}}$ since they trace the integrated mass along the line-of-sight which is roughly proportional to the density parameter of the Universe. Weak lensing by large scale structures has been measured by several teams as a coherent distortion

Send offprint requests to: F. Bernardeau,

e-mail: fbernard@spht.saclay.cea.fr

* Based on observations obtained at the Canada-FranceHawaii Telescope (CFHT) which is operated by the National Research Council of Canada (NRCC), the Institut des Sciences de l'Univers (INSU) of the Centre National de la Recherche Scientifique (CNRS) and the University of Hawaii (UH). field of distant galaxies over large angular distances (Bacon et al. 2000 and 2002; Hämmerle et al. 2002; Hoekstra et al. 2002; Kaiser et al. 2000; Maoli et al. 2001; Réfrégier et al. 2002; Rhodes et al. 2001; van Waerbeke et al. 2000, 2001 and 2002; Wittman et al. 200). The projected mass density reconstructed from the distortion field (i.e. the convergence field) can be used for non-Gaussian signatures searches, as shown in Bernardeau et al. (1997, hereafter BvWM97). This work and further studies (Jain \& Seljak 1997; van Waerbeke et al. 1999) have shown that the non-Gaussian properties of the convergence field can be used as a probe of the cosmological density parameter, with a weak dependence on the cosmological constant $\Omega_{\Lambda}$, provided that the redshift distribution of the sources is known.

However, a straight application of these theoretical considerations to real data sets turned out to be arduous. The convergence field has to be recovered from a mass reconstruction process which uses a continuous shear field obtained from a smoothed map of the discrete galaxy ellipticities. Unfortunately, survey topologies are generally complex and are altered by masked areas due to light scattering, bright stars, comet-like reflections, asteroid/airplane tracks, very bright galaxies, etc. Since the mask sizes cover a range of scales from a few arc-seconds to two degrees and are strongly anisotropic (for instance bright stars preferentially saturate along CCD columns), 
results of mass reconstruction in such data sets are not yet reliable. An alternative approach is the aperture mass applied to cosmic shear (Schneider et al. 1998), which allows the measurement of the skewness from the distortion field directly, bypassing the mass reconstruction process. So far, our attempts for measuring the skewness of the aperture mass lead to very noisy and insignificant results. Recently, Bernardeau et al. (2002, hereafter BvWM02) have proposed a new method using some specific patterns in the shear three-point function. This method has also the advantage to bypass the mass reconstruction process. Despite the complicated shape of the three-point correlation pattern, BvWM02 uncovered a specific geometrical property and demonstrated it can be used for the measurement of non-Gaussian features. Their detection strategy based on this method has been found to be robust, usable in patchy catalogs, and quite insensitive to the topology of the survey. In the following we apply this method to the VIRMOS-DESCART weak lensing survey done at the Canada-France-Hawaii Telescope, and we refer to our companion paper (BvWM02) for the technical details and the complete derivation of our estimator.

\section{Optimized 3-point correlation function applied to VIRMOS-DESCART data}

\subsection{Method}

Let us consider a triplet of galaxies at locations $\boldsymbol{x}_{1}, \boldsymbol{x}_{2}$ and $\boldsymbol{x}^{\prime}$, and their shear estimates $\boldsymbol{\gamma}\left(\boldsymbol{x}_{1}\right), \boldsymbol{\gamma}\left(\boldsymbol{x}_{2}\right)$ and $\boldsymbol{\gamma}\left(\boldsymbol{x}^{\prime}\right)$. BvWM02 introduced a 2D angular vector field (representing 2 components of the general shear 3-point correlation functions),

$\boldsymbol{\xi}_{3}\left(\boldsymbol{x}^{\prime}\right)_{d_{12}}=\left\langle\left(\boldsymbol{\gamma}\left(\boldsymbol{x}_{1}\right) \cdot \boldsymbol{\gamma}\left(\boldsymbol{x}_{2}\right)\right) \boldsymbol{\gamma}\left(\boldsymbol{x}^{\prime}\right)\right\rangle$

viewed as a function of $\boldsymbol{x}^{\prime}$ for a fixed $d_{12}=\left|\boldsymbol{x}_{2}-\boldsymbol{x}_{1}\right|$ distance. The ensemble average is taken over all possible pair orientation at fixed $d_{12}$. The two components of $\boldsymbol{\xi}_{3}$ are expected to scale like the square of the shear 2-point correlation function, $\xi_{2}\left(\boldsymbol{x}_{12}\right)=\left\langle\boldsymbol{\gamma}\left(\boldsymbol{x}_{1}\right) \cdot \boldsymbol{\gamma}\left(\boldsymbol{x}_{2}\right)\right\rangle$. Its sensitivity to the cosmological mean density $\Omega$ is similar to the convergence skewness $\left(\approx \Omega^{-0.8}\right)$. The way $\boldsymbol{\xi}_{3}\left(\boldsymbol{x}^{\prime}\right)$ varies as a function of $\boldsymbol{x}^{\prime}$ is in general complicated although asymptotic properties can be obtained analytically (see BvWM02). However, $\boldsymbol{\xi}_{3}\left(\boldsymbol{x}^{\prime}\right)_{d_{12}}$ has been found to be rather uniform and orthogonal to $\boldsymbol{x}_{1}-\boldsymbol{x}_{2}$ inside an elliptic area surrounding the segment joining $\boldsymbol{x}_{1}$ to $\boldsymbol{x}_{2}$. This pattern turns out to be robust against different cosmologies and smoothing scales, with an amplitude which can be related to the cosmological parameters. The results obtained by BvWM02 in synthetic catalogs suggested that present-day cosmic shear surveys were already large for a secure detection.

We considered the geometrical average inside this elliptical area $V_{\mathrm{Ell}}$,

$\overline{\boldsymbol{\xi}_{3}}\left(d_{12}\right)=\int_{\text {Ell. }} \frac{\mathrm{d}^{2} \boldsymbol{x}^{\prime}}{V_{\text {Ell. }}} \boldsymbol{\xi}_{3}\left(\boldsymbol{x}^{\prime}\right)_{d_{12}}$

where the points $\boldsymbol{x}_{1}$ and $\boldsymbol{x}_{2}$ are taken as the foci of the ellipse. Inside $V_{\mathrm{Ell}}, \boldsymbol{\xi}_{3}\left(\boldsymbol{x}^{\prime}\right)_{d_{12}}$ is weakly varying, and the two components of $\overline{\boldsymbol{\xi}_{3}}$ represent a vector whose average is orthogonal to the $\boldsymbol{x}_{1}-\boldsymbol{x}_{2}$ line. It means that only one of the two components of $\overline{\boldsymbol{\xi}_{3}}$ is non-zero, and in the following we call this component $\bar{\xi}_{3}{ }^{\mathrm{t}}$, which is the quantity we directly measure from the data. BvWM02 checked that realistic sources of noise, including the intrinsic ellipticity of galaxies, shape measurements errors, PSF correction and masking effects do not spoil the result. Final tests and validations were carried out with simulated catalogues containing as many galaxies as real data, with ellipticity distribution, PSF anisotropy and masking templates similar to the VIRMOS-DESCART sample (see BvWM02 for details). In all configurations the global signal to noise remains higher than 5 for scales between 30 arcsec to 5 arcmin.

\subsection{The VIRMOS-DESCART sample}

The VIRMOS-DESCART sample used in this work is part of the DESCART cosmic shear program ${ }^{1}$ which uses the VIRMOS photometric/imaging survey ${ }^{2}$ for wide field cosmic shear. It covers $11.5 \mathrm{deg}^{2}$ of CFH12K images spread over four uncorrelated fields. All data were obtained in $I$ band up to a limiting magnitude of $I_{A B}=24.5$ (within 5 arcsec aperture, $5-\sigma$ ), which is consistent with a mean source redshift of $z \simeq 0.9$ of the VIRMOS-DESCART sample (van Waerbeke et al. 2002). The data contain all observations used by van Waerbeke et al. (2001) plus new fields obtained in September and November 2000. All the images were processed as described in van Waerbeke et al. (2001) at the TERAPIX data center ${ }^{3}$. From an initial detection we build up a sample containing 1.6 millions objects. After masking and all galaxy selection processes, it reduces to 580000 galaxies, covering an effective area of $8.5 \mathrm{deg}^{2}$. Close-pairs with angular separation smaller than 10 arcsec can produce systematics and are rejected (see van Waerbeke et al. 2000).

Following standard notation, a noisy estimate of the shear per galaxy is obtained from

$e_{1}=e \cos (2 \theta), \quad e_{2}=e \sin (2 \theta)$

where $\theta$ is the angle between the major axis of the source galaxy and the $x$ axis, and the ellipticity $e=(1-r) /(1+r)$ depends on the galaxy axis ratio $r$. The galaxy ellipticities are computed according to the rules and weighting schemes given in Pen et al. (2002) and the PSF anisotropy corrections used in van Waerbeke et al. (2002). The statistical estimators for the binned two and three point functions are given by

$\begin{aligned} \xi_{2}\left(d_{i j}\right) & =\frac{\sum_{i j} w_{i} w_{j}\left(\boldsymbol{e}_{i} \cdot \boldsymbol{e}_{j}\right)}{\sum_{i j} w_{i} w_{j}} \\ \bar{\xi}_{3}^{\mathrm{t}}\left(d_{i j}\right) & =\frac{\sum_{i j k} w_{i} w_{j} w_{k}\left(\boldsymbol{e}_{i} \cdot \boldsymbol{e}_{j}\right) e_{k}^{(i j)}}{\sum_{i j k} w_{i} w_{j} w_{k}}\end{aligned}$

\footnotetext{
1 http://terapix.iap.fr/Descart

2 http://www.astrsp-mrs.fr

3 http://terapix.iap.fr
} 
where $e_{k}^{(i j)}$ is opposite to the component of the ellipticity of galaxy $k$ along the $\left(\boldsymbol{x}_{j}-\boldsymbol{x}_{i}\right)$-direction. The summations are made for pairs or triplets such that $d_{i j}=\left|\boldsymbol{x}_{i}-\boldsymbol{x}_{j}\right|$ is in the chosen bin. $\boldsymbol{x}_{k}$ lies within the ellipse defined by $\left|\boldsymbol{x}_{k}-\boldsymbol{x}_{i}\right|+\left|\boldsymbol{x}_{k}-\boldsymbol{x}_{j}\right|>1.1\left|\boldsymbol{x}_{i}-\boldsymbol{x}_{j}\right|$ and $w_{i}$ are weights associated to each galaxy according to the scheme discussed in BvWM02. We also consider in this Letter the decomposition of the two-point correlation function into $E$ and $B$ modes as described in Pen et al. (2002) which is used for residual systematics checks. In the following we write $\xi_{3}$ instead of $\bar{\xi}_{3}^{\mathrm{t}}$ in order to simplify the notation.

\subsection{The VIRMOS-DESCART 2 and 3-point correlation functions}

Figure 1 shows the estimated 2-point (top) and reduced 3 -point (bottom) correlation functions ( $\xi_{3}$ in units of $\xi_{2}^{2}$ ). Measurements have been made in regularly spaced bins of width 400 pixels (e.g. $1.3 \mathrm{arcmin}$ ). For comparison, the thick dotted lines shows the corresponding quantity measured in ray-tracing simulations for an open CDM model, the thick dashed line for a $\tau \mathrm{CDM}$ and the dot-dashed line for a $\Lambda \mathrm{CDM}$ model. The source redshift in these simulations is one, very close to our mean source redshift of 0.9 . In this plot the error bars are the measurement errors and do not include the cosmic variance (see BvWM02). The dot-dot-dashed line represents the signal corrected from the residual systematics (the $B$-mode contribution to the two-point correlation function subtracted from the $B$ mode contribution as discussed in Pen et al. 2002), while the solid line shows the total $(E+B)$ measured signal. The closeness of the two curves reveals the small amount of residual systematics still present in the 2-point correlation function.

A quality assessment of the 3-point function measurements can be done by studying the effect of PSF correction on the star 3-points function. The star shapes are measured from the standard procedure described in Kaiser et al. (1995). It can be thought as a star shear field that has to be subtracted off the measured galaxy shapes. One can then measure the 2- and 3-point correlation functions of the star field and compare them to the PSF corrected data set. This is presented in Fig. 2. The solid line in the top and bottom plots respectively show the star two and three point functions, which are compared to the same quantities measured on the corrected galaxies (dashed lines and dashed-dot line for the corrected $E$-mode 2-point function). The star two-point correlation function is significantly larger than the galaxy signal, but this is known not to be a problem for the twopoint function: Erben et al. (2001) and Bacon et al. (2001) have shown that the PSF correction can account for star anisotropies as large as 10\% (the stars rms anisotropy is $6 \%$ in our data) to a precision better than than one percent. This is also demonstrated in BvWM02 using cosmic shear simulated data (Moreover, the small amplitude of the $B$-mode is also an independent proof of the
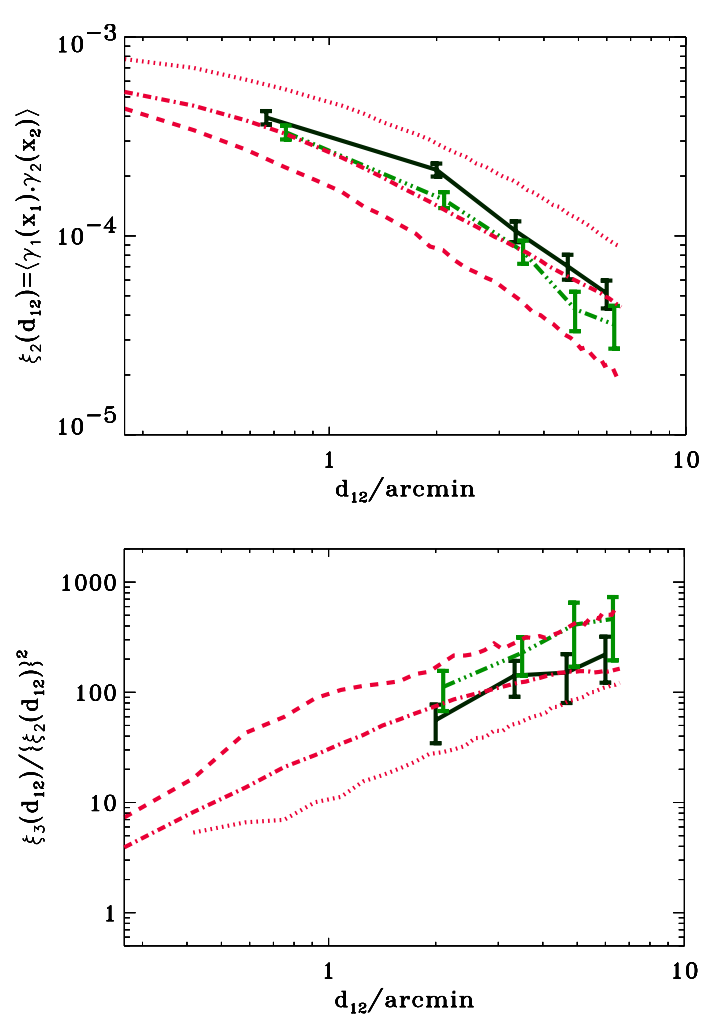

Fig. 1. Results for the VIRMOS-DESCART survey for the two point correlation function (top) and the reduced three point function (bottom). The solid line with error bars shows the raw results, when both the $E$ and $B$ contributions to the two-point correlation functions are included. The dot-dashed line with error bars corresponds to measurements where the contribution of the $B$ mode has been subtracted out from the two-point correlation function (but not from $\boldsymbol{\xi}_{3}$ there is no known way to do it). These measurements are compared to results obtained in $\tau \mathrm{CDM}$, OCDM and $\Lambda \mathrm{CDM}$ simulations (dashed, dotted and dot-dashed lines respectively). ( $\tau \mathrm{CDM}$, OCDM simulations are described in Jain et al. 2000; $\Lambda$ CDM simulation was provided by M. White.)

robustness of the correction). BvWM02 have shown that the correction scheme works also very well for the shear three-point function. However, we see that the situation for the three-point function (Fig. 2, bottom panel) is far more interesting than the two-point function: the correction is smaller (that is in principle more robust), and the angular dependence of the star three point function is totally different from the galaxy three-point function. If our three-point function signal were dominated by systematics it would likely not fit the expected signal as we can see from Fig. 1, but would be more similar to Fig. 2, bottom panel (solid line). Finally we have checked that cuts in magnitudes do not significantly change the results. Therefore, they are not produced by one single class of objects of the sample. We have thus several evidences that our signal is genuinely cosmological.

Another issue, not taken into account in the error bars, is the so-called cosmic variance, that is the amount by which such a signal can vary in finite size surveys. 

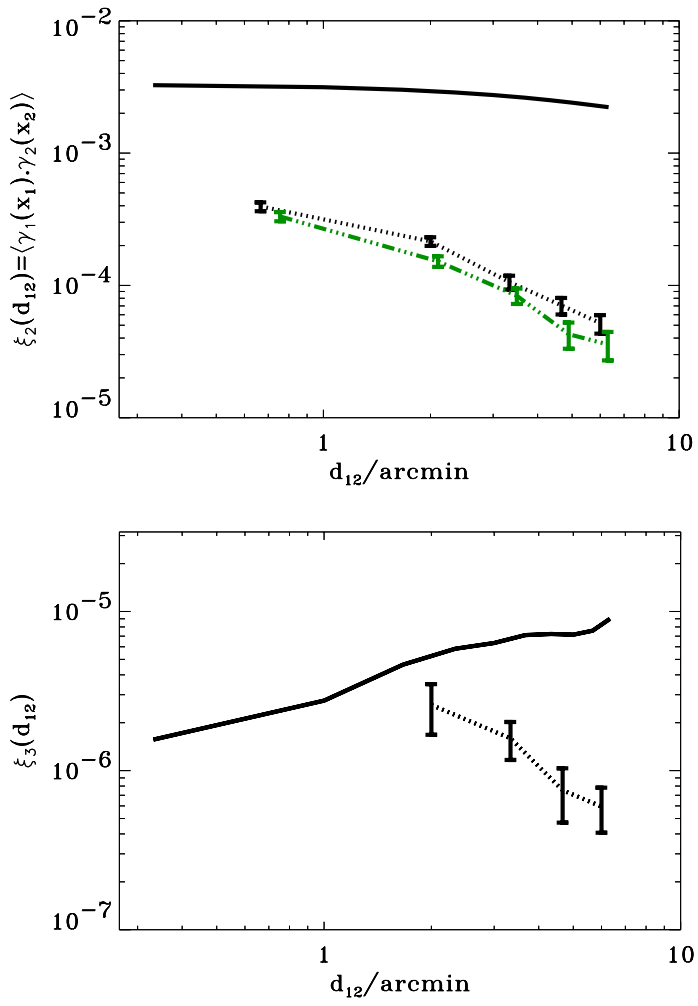

Fig. 2. Results for the VIRMOS-DESCART survey (dashed lines: when $E$ and $B$ mode contributions are included, dotdashed line when $B$ mode contributions are subtracted out from the 2-point correlation function) compared the properties of the star anisotropy field statistics (solid lines).

The complete theory of cosmic variance for such a survey is yet to be done, therefore we used several realizations of ray-tracing simulations to estimate it. Figure 3 shows the cosmic variance obtained from a set of 7 ray-tracing realizations of the open CDM model (Jain et al. 2000). Note that the error-bars in the different bins are correlated. A rough examination of the situation shows that while the cosmic variance dominates the error budget for the two-point function, observational errors and cosmic variance are of similar amplitude for the three-point function. From the examination of Fig. $1 \Lambda \mathrm{CDM}$ is clearly in better agreement with the data than the other two models, while $\tau \mathrm{CDM}$ is marginally acceptable.

\section{Discussion}

The result shown in Fig. 1 is the first detection of nonGaussian features in a cosmic shear survey. The signal is detected with a 2.4- $\sigma$ confidence level on 4 independent bins which gives a 4.9- $\sigma$ global confidence level. Such a result opens the route for breaking the degeneracy between $\Omega_{\mathrm{m}}$ and $\sigma_{8}$ in a way which is independent on assumptions beyond the solely hypothesis that large-scale structures grows from gravitational instability of an initial Gaussian field. We note that the amplitude of the reduced 3-point correlation function exhibits an angular dependence which is in agreement with theoretical

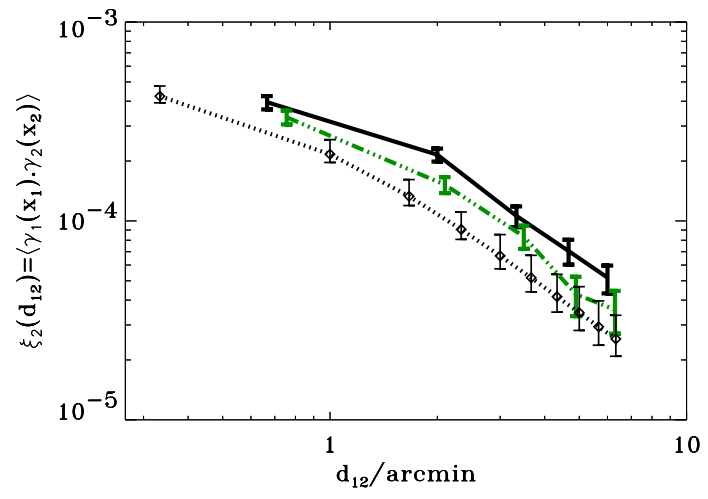

expectations. It supports the interpretation of these results as genuine effects of the gravitational dynamics.

The signal is however still too noisy to provide reliable information on cosmological parameters. Moreover, several obstacles have yet to be overcome. It is first important to understand to which level the 3-points measurements are contaminated by systematics. This can be done through consistency checks yet to be invented (a statistic which cancels the signal in a non-trivial way, like the 45 degrees rotation test for the aperture mass will have to be found for the three-point function). We have already checked with the anisotropy of the stars that our signal is unlikely to be dominated by systematics since it would otherwise exhibits a totally different angular dependence. In the next stages, the scientific interpretation of the three-point function measurements will require a significant improvement over several issues:

- The knowledge of the redshift distribution of the sources is crucial for the third order statistics (see BvWM97);

- The source clustering effect might bias the measurement is a significant way (Bernardeau 1998; Hamana et al. 2002) if the width of the source distribution is too large;

- Intrinsic alignment of galaxies have a completely unknown effect on the non-Gaussian properties of the shear field. 
Resolving these issues will require progress from both the theoretical/simulation side and from the observations, which are already on their way.

Acknowledgements. We thank the VIRMOS and Terapix teams who got and processed the VIRMOS-DESCART data, B. Jain and M. White for the use of their ray-tracing simulations as well as T. Hamana for useful comments. This work was supported by the TMR Network "Gravitational Lensing: New Constraints on Cosmology and the Distribution of Dark Matter" of the EC under contract No. ERBFMRX-CT97-0172. FB thanks IAP for hospitality.

\section{References}

Bacon, D., Réfrégier, A., \& Ellis, R. S. 2000, MNRAS, 318, 625 Bacon, D., Massey, R., Réfrégier, A., \& Ellis, R. S. 2002, preprint [astro-ph/0203134]

Bacon, D., Réfrégier, A. D., Clowe, D., \& Ellis, R. S. 2001, MNRAS, 325, 1065

Bernardeau, F. 1992, ApJ, 392, 1

Bernardeau, F. 1994, A\&A, 433, 1

Bernardeau, F. 1998, A\&A, 338, 375

Bernardeau, F., van Waerbeke, L., \& Mellier, Y. 1997, A\&A, 322, 1 (BvWM97)

Bernardeau, F., van Waerbeke, L., \& Mellier, Y. 2002, preprint [astro-ph/0201029] (BvWM02)

Bouchet, F. R., Juszkiewicz, R., Colombi, S., \& Pellat, R. 1992, ApJL, 394, L5

Erben, T., van Waerbeke, L., Bertin, E., Mellier, Y., \& Schneider, P. 2001, A\&A, 366, 717
Hamana, T., Colombi, S., Thion, A., et al. 2002, MNRAS, 330, 365

Hämmerle, H., Miralles, J.-M., Schneider, P., et al. 2002, A\&A, 385,743

Hoekstra, H., Yee, H., Gladders, M. D., et al. 2002, preprint [astro-ph/0202285]

Jain, B., \& Seljak, U. 1997, ApJ, 484, 560

Jain, B., Seljak, U., \& White, S. D. M. 2000, ApJ, 530, 547

Kaiser, N., Wilson, G., \& Luppino, G. 2000, preprint [astro-ph/0003338]

Kaiser, N., Squires, G., \& Broadhusrt, T. 1995, ApJ, 449, 460

Maoli, R., van Waerbeke, L., Mellier, Y., et al. 2001, A\&A, 368,766

Peebles, P. J. E., The Large-Scale Structure of the Universe (Princeton University Press), 1980

Pen, U. L., van Waerbeke, L., \& Mellier, Y. 2002, ApJ, 567, 31

Rhodes, J., Réfrégier, A., \& Groth, E. J. 2001, ApJ, 536, 79

Réfrégier, A., Rhodes, J., \& Groth, E. J. 2002, preprint [astro-ph/0203131]

Schneider, P., van Waerbeke, L., Jain, B., \& Kruse, G. 1998, MNRAS, 296, 873

van Waerbeke, L., Bernardeau, F., \& Mellier, Y. 1999, A\&A, 342,15

van Waerbeke, L., Mellier, Y., Erben, T., et al. 2000, A\&A, 358,30

van Waerbeke, L., Mellier, Y., Radovich, M., et al. 2001, A\&A, 374,751

van Waerbeke, L., Mellier, Pelló, R., et al. 2002, preprint [astro-ph/0202503]

Wittman, D., Tyson, J. A., Kirkman, D., Dell'Antonio, I., \& Bernstein, G. 2000, Nature, 405, 143 